ARTIGO ORIGINAL

\title{
Caracterizações anatômicas e físicas da madeira de pau-mulato (Calycophyllum spruceanum)
}

\author{
Anatomical and physical characterizations of Calycophyllum spruceanum \\ wood
}

Jaily Kerller Batista de Andrade ${ }^{1}$ (D), Marina Donária Chaves Arantes ${ }^{2}$ (D), Juarez Benigno Paes ${ }^{1}$ (D), José Tarcísio da Silva Oliveira ${ }^{1}$ (D), Sandra Bezerra da Silva ${ }^{3}$ (D), Anderson Vasconcelos Firmino ${ }^{1}$ (D)

${ }^{1}$ Universidade Federal do Espírito Santo - UFES, Jerônimo Monteiro, ES, Brasil

${ }^{2}$ Universidade Federal de São João Del Rei - UFSJ, Sete Lagoas, MG, Brasil

${ }^{3}$ Universidade Federal do Acre - UFAC, Rio Branco, AC, Brasil

Como citar: Andrade, J. K. B., Arantes, M. D. C., Paes, J. B., Oliveira, J. T. S., Silva, S. B., \& Firmino, A. V. (2020). Caracterizações anatômicas e físicas da madeira de pau-mulato (Calycophyllum spruceanum). Scientia Forestalis, 48(126), e2897. https://doi.org/10.18671/scifor.v48n126.02

\begin{abstract}
Resumo
Plantios homogêneos necessitam de desbastes, para a obtenção de madeira de melhor qualidade, sendo necessário um destino para as árvores retiradas, a fim de se obter maior rendimento do empreendimento florestal. Assim, o objetivo do estudo foi determinar as características anatômicas e físicas da madeira jovem de pau-mulato (Calycophyllum spruceanum Benth.) no sentido longitudinal do tronco. O material foi coletado em um plantio experimental da Embrapa Acre, localizado no município de Rio Branco, Acre, composto por 300 árvores com idade de seis anos, plantadas em espaçamento de $3 \times 2 \mathrm{~m}$. Para o estudo, foram demarcadas aleatoriamente cinco árvores, das quais foram mensuradas as alturas total e comercial e retirados discos com espessura de $3 \mathrm{~cm}$, nas posições 0, 25, 50, 75 e $100 \%$ da altura comercial (diâmetro mínimo de $5 \mathrm{~cm}$ ), e um suplementar na altura do peito, tomada a 1,30 m da superfície do solo. Foram analisadas as características anatômicas, massa específica básica e a retratibilidade da madeira ao longo do tronco. As propriedades anatômicas e físicas da madeira variaram no sentido longitudinal, o que permitiu o entendimento do seu comportamento (variação) ao longo do tronco, na idade amostrada, uma vez que, pouco se conhece sobre as características anatômicas e físicas do pau-mulato proveniente de reflorestamento. Assim, os resultados obtidos contribuirão com o conhecimento científico, tornando uma ferramenta considerável para valorização e comercialização da madeira.
\end{abstract}

Palavras-chave: Espécie florestal tropical; Variação longitudinal; Anatomia da madeira; Propriedades físicas da madeira.

\begin{abstract}
Homogeneous plantations need thinning, to obtain better quality wood, a destination for the trees removed, in order to achieve optimum performance of the enterprise. Thus, the study aimed to determine the anatomic and physical characteristics of Calycophyllum spruceanum Benth. wooden young in longitudinal direction of the trunk. The material for the study was collected in an experimental planting of Embrapa Acre, located in the municipality of Rio Branco, Acre, Brazil, composed by 300 trees with six years old, and planted in spacing $3 \times 2 \mathrm{~m}$. In that five trees chosen randomly were demarcated, of which were measured the total and commercial heights and removed disks whit $3 \mathrm{~cm}$ thick, in the positions 0, 25, 50, 75 and 100\% (minimum $5 \mathrm{~cm}$ ), and a supplement one was take in chest height, taking $1.30 \mathrm{~m}$ from the surface of the soil. Anatomical characteristics, specific gravity basic and shrinkage of wood along the trunk were analyzed. The anatomical and physical properties of wood varied longitudinally which enabled the understanding of your behavior (variation) along of the trunk, in the age sampled, once little
\end{abstract}

Fonte de financiamento: Coordenação de Aperfeiçoamento de Pessoal de Nível Superior (CAPES) - Código de Financiamento 001.

Conflito de interesse: Nada a declarar.

Autor correspondente: jbp2@uol.com.br

Recebido: 18 maio 2018

Aceito: 27 junho 2019.

Editor: Paulo Henrique Müller Silva

(c) (1) Este é um artigo publicado em acesso aberto (Open Access) sob a licença Creative Commons Attribution, que permite uso, distribuição e reprodução cc) em qualquer meio, sem restrições desde que o trabalho original seja corretamente citado. 
is known about the anatomical and physical characteristics of the studied forestry specie from reforestation. Thus, the results obtained will contribute with scientific knowledge, making a considerable tool for valorization and wood commercialization.

Keywords: Forestry tropical specie; Longitudinal variation; Wood anatomy; Physical properties of wood.

\section{INTRODUÇÃO}

A silvicultura brasileira se desenvolveu com o estudo de espécies florestais exóticas, principalmente as do gênero Eucalyptus e Pinus. No entanto, outras vêm sendo plantadas e pesquisadas para a produção madeireira. Neste contexto, espécies nativas de rápido crescimento como o pau-mulato (Calycophyllum spruceanum Benth.) podem contribuir com o suprimento da demanda, principalmente pelo seu porte, uma vez que, segundo Lorenzi (2002) pode atingir altura de $20-40 \mathrm{~m}$ e diâmetro de $30-40 \mathrm{~cm}$, tronco retilíneo e com poucas ramificações. Mas, o plantio homogêneo dessa espécie, necessita de desbastes, a fim de conduzir a floresta para a obtenção de um produto final (madeira) de melhor qualidade. Desta forma, é necessário um destino para a madeira proveniente das intervenções realizadas no plantio.

A madeira de pau-mulato é moderadamente pesada, dura, compacta, de boa trabalhabilidade e resistente à deterioração biológica (Lorenzi, 2002; Sotelo Montes et al., 2007; Guedes, 2015). Além disso, a constituição anatômica, como a presença de fibras com paredes moderadamente espessas associada à escassez de tecidos parenquimáticos lhe atribuem resistência mecânica e aumento da massa específica (Baldin \& Marchiori, 2015). Com essas características, espécies de Calycophyllum são comumente empregadas em marcenarias na fabricação de móveis e na construção civil (Boivin-Chabot et al., 2004; Sotelo Montes et al., 2007).

O conhecimento da estrutura e organização dos elementos anatômicos que compõem o lenho, além de possibilitar a identificação de uma espécie florestal, permite a compreensão do comportamento da madeira perante operações de secagem, desdobro e impregnação. No entanto, o material é muito heterogêneo, podendo ocorrer variações em suas propriedades nas direções radial, tangencial e longitudinal e ao longo do tronco (Panshin \& De Zeeuw, 1980).

Uma das principais propriedades da madeira e de fácil determinação é a massa específica básica, a qual é utilizada como parâmetro de qualidade do material, pois lenhos menos densos possuem mais tecidos parenquimáticos, ou fibras de paredes finas, influenciando na sua resistência mecânica. Enquanto os mais compactos, geralmente são mais resistentes, escuros e possuem maior contração volumétrica. Assim, essa característica, apesar de ser um bom indicativo da rigidez, pode ser influenciada pelas variações existente entre árvores de uma mesma espécie, idade, qualidade de sítio e pelas condições ambientais (Paula, 2005; Sotelo Montes et al., 2007).

Entretanto, a massa específica não deve ser considerada um índice isolado de qualidade da madeira. Sua composição química primária e secundária (quantidade e classes de extrativos e localização na célula) e, as características anatômicas também são indicadores de qualidade (Pimenta et al., 2010), por influenciarem as demais propriedades da madeira. Desta forma, o estudo anatômico aliado ao comportamento das propriedades físicas da madeira são parâmetros essenciais para estabelecer o melhor uso desse material (Burger \& Richter, 1991; Trianoski et al., 2013).

Associado a tais fatos, as variações nas dimensões da madeira, ocasionadas pelo aumento ou diminuição da umidade são características importantes e devem ser consideradas quando da escolha do material, pois quanto menor a alteração volumétrica, juntamente com o baixo coeficiente de anisotropia, melhor será a qualidade da madeira, principalmente quando se deseja utilizá-la na fabricação de móveis, pisos e lambris (Trianoski et al., 2013).

Em decorrência da falta de informações sobre a qualidade da madeira jovem de pau-mulato, proveniente de reflorestamento, das variações das propriedades tecnológicas ocorridas dentro da árvore e seus efeitos na utilização da mesma, este estudo teve como objetivo determinar as 
características anatômicas e físicas da madeira jovem de pau-mulato (Calycophyllum spruceanum) no sentido longitudinal do tronco.

\section{MATERIAL E MÉTODOS}

\section{Descrição do plantio, amostragem e determinação do volume de madeira}

A madeira de Calycophyllum spruceanum para o estudo foi coletada em uma parcela experimental da Embrapa Acre, localizada no município de Rio Branco, Acre; composta por 300 árvores com idade de seis anos, plantadas em espaçamento de $3 \times 2 \mathrm{~m}$. O plantio compreende uma área total de 1.200 ha e está localizado entre as coordenadas geográficas de $10^{\circ} 01^{\prime} 22^{\prime \prime}$ e $10^{\circ} 04^{\prime} 14^{\prime \prime}$ de latitude sul e de $67^{\circ} 40^{\prime} 3^{\prime \prime}$ e $67^{\circ} 42^{\prime} 43^{\prime \prime}$ de longitude a oeste de Greenwich.

Para o estudo, foram demarcadas aleatoriamente cinco plantas (diâmetro médio de $8,90 \mathrm{~cm}$ e altura total de $9,90 \mathrm{~m}$ ), as quais estavam livres de ataque de insetos ou qualquer tipo de injúria. Após a derrubada, foram mensuradas as alturas total e comercial das mesmas e retirados discos com espessura de $3 \mathrm{~cm}$, nas posições $0,25,50,75$ e $100 \%$ da altura comercial (diâmetro mínimo de $5 \mathrm{~cm}$ ), e um suplementar na altura do peito (DAP), tomada a 1,30 m da superfície do solo.

Os discos foram identificados e levados ao pátio da Embrapa Acre para secagem ao ar e, posteriormente transportados para o Departamento de Ciências Florestais e da Madeira, Universidade Federal do Espírito Santo, localizado em Jerônimo Monteiro, ES, onde foram realizados os estudos.

Os volumes dos troncos com e sem casca foram estimados pelo método de Smalian, como descrito por Campos \& Leite (2002). A determinação do volume de madeira (metros cúbicos e em porcentagem) foi realizada por diferença entre o volume do tronco (com casca) e o volume de casca.

\section{Descrição anatômica}

Para o estudo anatômico da madeira, foram avaliadas as características macroscópicas e microscópicas. Para tanto, adotaram-se as determinações da Comissión Panamericana de Normas Técnicas (1974) e International Association of Wood Anatomists (2007).

A descrição macroscópica foi realizada com uso de uma lente de 10x de aumento. Os elementos anatômicos observados foram as camadas de crescimento, poros, raios e tipo de parênquima.

Para a descrição microscópica, foram produzidos corpos de prova com dimensões de $1,0 \times 1,5 \times 2,0 \mathrm{~cm}$ (tangencial $\times$ radial $\times$ longitudinal), obtidos do cerne periférico dos discos, tomados nas posições $0,25,50,75 \%, 100 \%$ da altura comercial e no DAP. As amostras foram amolecidas em água fervente, fixadas em micrótomo de deslize e produzidos cortes histológicos em cada uma das seções, com espessura de 14 a $20 \mu \mathrm{m}$.

A mensuração anatômica foi realizada com um sistema de aquisição de imagens composto de câmera acoplada a um microscópio e com o uso do software Image-Pro Plus para análise das imagens. Os elementos anatômicos avaliados foram os vasos (diâmetro e frequência), fibras (comprimento, largura, diâmetro do lume e espessura da parede) e raios (altura, largura e frequência), todas as mensurações com 20 observações.

A mensuração das fibras foi realizada com a dissociação dos elementos anatômicos que compõem a madeira, pelo uso da técnica de maceração, seguindo a metodologia proposta por Nicholls e Dadswell, descrita por Ramalho (1987), em que foram preparados pequenos palitos de madeira no sentido radial, os quais foram alocados em tubos de ensaios contendo solução macerante (nítrico-acético, 1:5). Os tubos foram lacrados e levados para estufa à temperatura de $60^{\circ} \mathrm{C}$ por um período de 48 horas. O macerado obtido foi lavado com água destilada e colorido com safranina $1 \%$ para o preparo de lâminas temporárias. As estruturas foram visualizadas em microscópio com câmera fotográfica acoplada e retirada das fotomicrografias. 
Massa específica básica e retratibilidade da madeira

A massa específica básica e retratibilidade da madeira foram determinadas ao longo da altura comercial do tronco. Em cada disco, foram retirados corpos de prova obtidos do cerne periférico, com dimensões $2 \times 2 \times 3 \mathrm{~cm}$ (radial x tangencial x longitudinal).

Para a determinação da massa específica básica, foram seguidas as especificações da Norma Brasileira Regulamentadora - NBR 11941 (Associação Brasileira de Normas Técnicas, 2003). A retratibilidade da madeira foi determinada de acordo com a NBR 7190 (Associação Brasileira de Normas Técnicas, 1997), ao utilizarem as mesmas amostras destinadas à determinação da massa específica básica.

\section{Análise estatística dos dados}

$\mathrm{Na}$ avaliação das características anatômicas quantitativas, massa específica básica e retratibilidade da madeira foi utilizado o delineamento experimental inteiramente casualizado. Para todas as variáveis foi verificada a normalidade dos dados pelo teste de Lilliefors e a homogeneidade das variâncias pelo teste de Cochran.

Os valores obtidos foram submetidos à análise de variância e quando detectadas diferenças significativas entre os tratamentos (teste $F, p \leq 0,05$ ), as médias foram comparadas pelo teste de Scott-Knott $(p \leq 0,05)$.

\section{RESULTADOS E DISCUSSÃO}

\section{Características gerais e descrição macroscópica da madeira}

As árvores de pau-mulato possuíram DAP com casca de $8,90 \mathrm{~cm}$ e sem casca de e $8,68 \mathrm{~cm}$, alturas comerciais e totais médias de 5,68 e 9,90 m, respectivamente, volume de madeira de $0,024 \mathrm{~m}^{3}$, correspondente a $95,09 \%$ do total do tronco e incremento médio anual (IMA), em diâmetro de 1,48 cm ano-1. Para árvores de 5,3 anos, provenientes da Amazônia Peruana, Ugarte-Guerra \& Domínguez-Torrejón (2010) encontraram alturas totais de 8,7 a 12,5 m. Ugarte-Guerra \& Domínguez-Torrejón (2010) e Guedes et al. (2012), para árvores com idade de 5 a 11 anos, que cresceram em áreas de sistemas agroflorestais no Amapá, e concluíram, com base no IMA, que a espécie possui elevada capacidade de crescimento.

A madeira de Calycophyllum spruceanum possui textura fina com grã direita, é moderadamente dura ao corte manual no plano transversal e de boa trabalhabilidade, lisa ao tato, brilho moderado, cheiro e gosto imperceptíveis. Possui massa específica básica média de $0,636 \mathrm{~g} \mathrm{~cm}^{-3}$, sendo considerada madeira de massa moderada.

Possui parênquima axial ausente, mesmo sob lente de 10x de aumento; camadas de crescimento distintas, demarcadas por zonas fibrosas, com células dos raios alargadas na transição entre o lenho inicial e tardio, havendo a presença de fibras estreitas no lenho outonal. Poros pequenos e muito numerosos, somente visíveis sob lente de 10x.

As características observadas estão compatíveis com o estudo de Gomez et al. (2006) que descrevem o lenho de pau-mulato como de textura fina, lisa, brilho moderado, grã direita, cheiro e gosto imperceptíveis, poros solitários e múltiplos que variam de pequenos a médios e camadas de crescimento delimitadas por zonas fibrosa.

\section{Descrição microscópica da madeira}

Vasos: em porosidade difusa uniforme, de seção ovalada, com diâmetro tangencial pequeno $(62,33 \mu \mathrm{m})$; poros solitários e múltiplos de dois (Figura 1A e B), arranjo radial predominantemente. A frequência média é muito numerosa $\left(42,57\right.$ vasos $\left.\mathrm{mm}^{-2}\right)$. Possui elementos vasculares com presença de apêndices em suas extremidades (Figura 2B). 


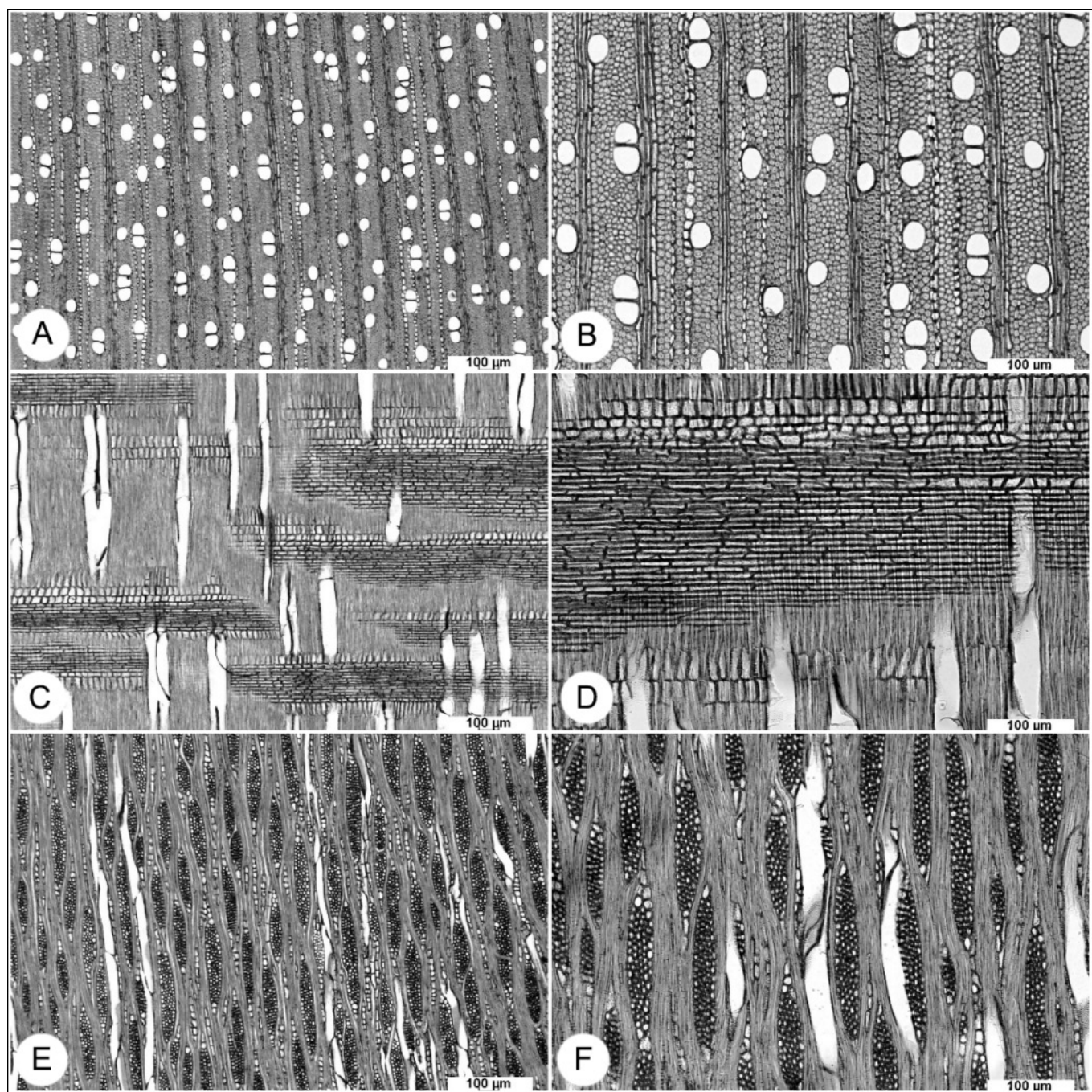

Figura 1. Fotomicrografias do lenho de Calycophyllum spruceanum com seis anos de idade. Seção transversal com poros/vasos solitários e múltiplos de dois, frequentes (A); mesma seção evidenciando os poros ovalados (B); Seção radial indicando os raios heterogêneos (C); mesma seção destacando as células eretas, quadradas e procumbentes (D); seção tangencial mostrando os raios não estratificados (E); mesma seção, com raios multisseriados (F).

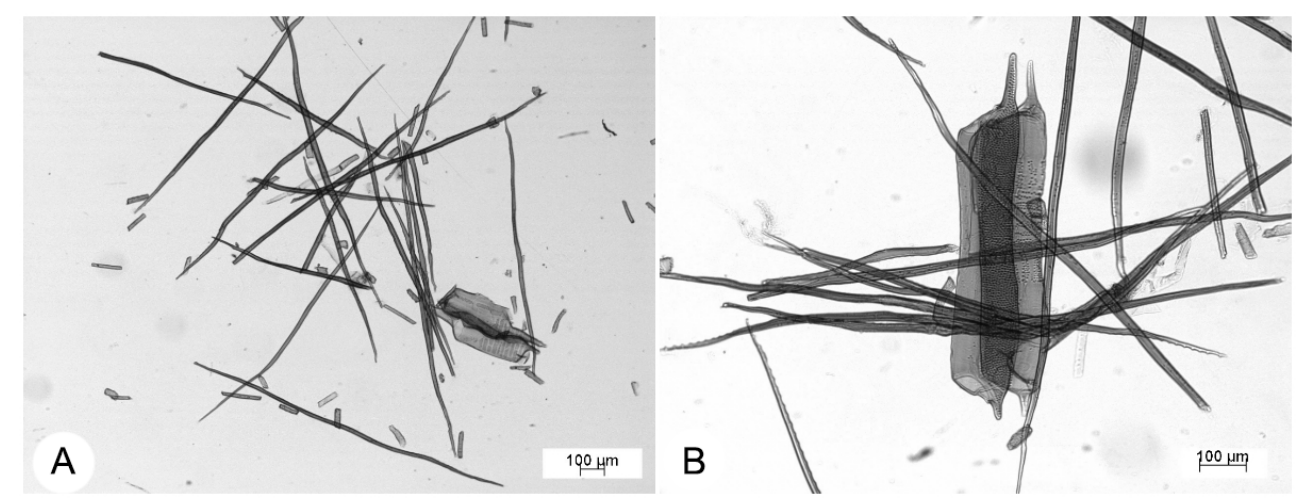

Figura 2. Fibras libriformes (A); vasos com apêndices nas extremidades (B).

Raios: Visíveis a olho nu no plano transversal. Heterogêneos, composto por células eretas, quadradas e procumbentes (Figura 1C e D). Exclusivamente multisseriados, não estratificados (Figura 1E e F). São finos $(37,46 \mu \mathrm{m})$, extremamente baixos $(0,46 \mathrm{~mm})$ e pouco frequentes $\left(11,14\right.$ raios $\left.\mathrm{mm}^{-1}\right)$. 
Fibras: libriformes (Figura 2A), curtas $(1349,57 \mu \mathrm{m})$, com paredes delgadas a espessas $(5,81 \mu \mathrm{m})$.

A descrição anatômica da espécie Calycophyllum spruceanum corrobora com os resultados mencionados por Baldin \& Marchiori (2015), os quais citam que a mesma possui raios heterogêneos, presença de células eretas, quadradas e procumbentes; vasos em porosidade difusa, solitários e em cachos, menos comumente; parênquima axial ausente; Fibras libriformes, curtas, com paredes delgadas a espessas.

\section{Características dos vasos}

Os valores médios e o coeficiente de variação dos parâmetros relacionados ao diâmetro e frequência de vasos da madeira de Calycophyllum spruceanum nas posições longitudinais do tronco, estão apresentados na Tabela 1.

Tabela 1. Valores médios do diâmetro e frequência de vasos da madeira de Calycophyllum spruceanum no sentido longitudinal, com seis anos de idade.

\begin{tabular}{cccccccc}
\hline \multirow{2}{*}{ Parâmetro } & \multicolumn{9}{c}{ Posição longitudinal (\%) } & Média \\
\cline { 2 - 7 } & $\mathbf{0}$ & DAP & $\mathbf{2 5}$ & $\mathbf{5 0}$ & $\mathbf{7 5}$ & $\mathbf{1 0 0}$ & Geral \\
\hline \multirow{2}{*}{ Diâmetro $(\mu \mathrm{m})$} & $49,33 \mathrm{c}$ & $61,66 \mathrm{~b}$ & $62,53 \mathrm{~b}$ & $68,32 \mathrm{a}$ & $68,52 \mathrm{a}$ & $63,65 \mathrm{~b}$ & 62,33 \\
& $(16,06)$ & $(16,70)$ & $(19,53)$ & $(18,55)$ & $(19,23)$ & $(25,16)$ & $(19,74)$ \\
Frequência & $39,97 \mathrm{~b}$ & $40,99 \mathrm{~b}$ & $44,57 \mathrm{a}$ & $41,51 \mathrm{~b}$ & $42,28 \mathrm{~b}$ & $46,08 \mathrm{a}$ & 42,57 \\
$\left(\mathrm{n}^{\circ} \mathrm{mm}^{-2}\right)$ & $(21,49)$ & $(16,29)$ & $(16,90)$ & $(21,19)$ & $(19,65)$ & $(24,17)$ & $(20,25)$ \\
\hline
\end{tabular}

$\mathrm{DAP}=$ diâmetro à altura do peito. Valores entre parênteses correspondem ao coeficiente de variação. Médias seguidas pela mesma letra na linha, não diferem (Scott-Knott, $p>0,05$ ).

De modo geral, o diâmetro dos vasos foi crescente, da base até a posição $75 \%$ da altura comercial da árvore, tendo leve decréscimo no topo (100\%). Observa-se que a espécie possui vasos de maiores diâmetros tangenciais nas posições 50, 75 e 100\% do tronco, sendo destacada a posição $75 \%$ por possui maior valor. A mesma não diferiu estatisticamente da posição 50\%. O menor diâmetro vascular foi encontrado na base das árvores (altura de corte).

Para a madeira de paricá (Schizolobium amazonicum) com idades de 5, 7, 9 e 11 anos, Vidaurre et al. (2018) observaram que o diâmetro dos vasos aumentou da base para 50\%, do comprimento do tronco, tendo, a partir desta posição um pequeno decréscimo, sem diferença estatística entre a amostragem realizada a $100 \%$ da altura comercial. No entanto, Gil et al. (2018) observaram, para a madeira de teca (Tectona grandis) obtida de desbaste, aos 7 anos, que o diâmetro dos vasos aumentou da base para o topo.

Em estudo realizado sobre a variação das dimensões dos elementos anatômicos da madeira de Eucalyptus grandis aos sete anos de idade, Rocha et al. (2004) relataram um padrão de variação semelhante para o diâmetro tangencial dos vasos. Os autores relatam que o maior valor de diâmetro de vaso foi obtido na posição $75 \%$ e o menor na base. No entanto, Paulino \& Lima (2018), para a madeira de clones de Eucalyptus urophylla, com oito anos de idade, observaram que a posição na altura da árvore diferiu apenas quanto à frequência dos vasos.

Segundo Maranho et al. (2006), mudanças nas dimensões das células estão correlacionadas com mudanças correspondentes na organização da parede celular, cuja alteração mais importante está associada ao ângulo de orientação das microfibilas presentes na camada $\mathrm{S}_{2}$ da parede secundária.

Nota-se que os coeficientes de variação, para o diâmetro dos vasos, observados nas seis posições, foram menor na base (altura de corte) e maior no topo (altura comercial da árvore) indicando uma variação mediana (menor que 30\%). Isso evidencia a pouca heterogeneidade entre os elementos anatômicos mensurados nas posições ao longo do tronco.

As médias dos tratamentos (posições longitudinais no tronco) para a frequência de vasos também diferiram estatisticamente pelo teste $F(p<0,05)$. Nas posições 25 e $100 \%$, foram superiores às demais, diferindo daquelas das posições $0, \mathrm{DAP}, 50$ e $75 \%$. O coeficiente de variação indicou que a frequência de vasos foi pouco heterogênea entre as posições. 
No entanto, Vidaurre et al. (2018) observaram que a frequência dos vasos do paricá decresceu da base para 50\%, do comprimento do tronco, tendo, a partir desta posição um pequeno decréscimo, sem diferença estatística entre a amostragem realizada a $100 \%$ da altura comercial. Para a madeira de teca, Gil et al. (2018) observaram que a frequência dos vasos decresceu da base até $25 \%$, e a partir de então, foi crescente.

De acordo com Bamber et al. (1982), o diâmetro dos vasos é inversamente proporcional à frequência. No entanto, a madeira do pau-mulato obteve maior frequência de vasos nas posições com maiores diâmetros tangenciais. Esse comportamento também foi observado por Gil et al. (2018) para a madeira de teca de sete anos.

Alterações na frequência, diâmetro e disposição dos vasos, segundo Tomazello Filho (1987), são explicadas pela necessidade das plantas aumentarem sua capacidade de transportar substâncias no sentido ascendente, ao passo que se processa o seu crescimento e aumento de suas dimensões.

\section{Características das fibras}

Nota-se que houve diferença entre as médias dos parâmetros relacionados às dimensões das fibras (Tabela 2). Observa-se que os valores de comprimento de fibra nas posições DAP, 25 e $50 \%$ obtiveram maiores valores e nas posições 0,75 e $100 \%$, os menores. Os coeficientes de variação referentes ao comprimento das fibras são considerados medianos. Gil et al. (2018) também observaram decréscimo no comprimento das fibras ao longo do tronco de árvores de teca com sete anos.

Tabela 2. Valores médios dos parâmetros mensurados das fibras da madeira de Calycophyllum spruceanum no sentido longitudinal, com seis anos de idade

\begin{tabular}{cccccc}
\hline Posição (\%) & $\begin{array}{c}\text { Comprimento } \\
(\boldsymbol{\mu m})\end{array}$ & $\begin{array}{c}\text { Largura } \\
(\boldsymbol{\mu m})\end{array}$ & $\begin{array}{c}\text { Diâmetro do } \\
\text { Lume } \\
(\boldsymbol{\mu m})\end{array}$ & $\begin{array}{c}\text { Espessura da } \\
\text { Parede } \\
(\boldsymbol{\mu m})\end{array}$ & $\begin{array}{c}\text { Fração } \\
\text { Parede } \\
(\%)\end{array}$ \\
\hline 0 & $1219,51 \mathrm{~b}$ & $20,93 \mathrm{a}$ & $8,88 \mathrm{a}$ & $6,03 \mathrm{a}$ & $57,98 \mathrm{a}$ \\
& $(28,20)$ & $(14,84)$ & $(27,18)$ & $(15,66)$ & $(12,57)$ \\
DAP & $1459,79 \mathrm{a}$ & $20,09 \mathrm{~b}$ & $8,27 \mathrm{~b}$ & $5,91 \mathrm{a}$ & $59,09 \mathrm{a}$ \\
& $(15,74)$ & $(12,51)$ & $(22,15)$ & $(12,44)$ & $(9,55)$ \\
25 & $1447,40 \mathrm{a}$ & $20,11 \mathrm{~b}$ & $8,18 \mathrm{~b}$ & $5,97 \mathrm{a}$ & $59,49 \mathrm{a}$ \\
& $(17,20)$ & $(14,68)$ & $(25,67)$ & $(17,69)$ & $(12,33)$ \\
50 & $1424,53 \mathrm{a}$ & $20,88 \mathrm{a}$ & $8,97 \mathrm{a}$ & $5,96 \mathrm{a}$ & $57,27 \mathrm{~b}$ \\
& $(14,88)$ & $(13,16)$ & $(25,83)$ & $(17,70)$ & $(14,98)$ \\
75 & $1269,15 \mathrm{~b}$ & $20,10 \mathrm{~b}$ & $9,06 \mathrm{a}$ & $5,52 \mathrm{~b}$ & $55,55 \mathrm{~b}$ \\
& $(16,60)$ & $(17,80)$ & $(33,64)$ & $(19,12)$ & $(16,91)$ \\
& $1277,04 \mathrm{~b}$ & $19,58 \mathrm{~b}$ & $8,68 \mathrm{a}$ & $5,46 \mathrm{~b}$ & $55,95 \mathrm{~b}$ \\
& $(19,67)$ & $(13,53)$ & $(24,29)$ & $(16,83)$ & $(13,33)$ \\
& 1349,57 & 20,28 & 8,67 & 5,81 & 57,55 \\
Média Geral & $(18,78)$ & $(14,52)$ & $(26,91)$ & $(16,59)$ & $(13,39)$ \\
\hline
\end{tabular}

DAP = diâmetro à altura do peito. Valores entre parênteses correspondem ao coeficiente de variação. Médias seguidas pela mesma letra na coluna, não diferem (Scott-Knott, $p>0,05$ ).

Para o comprimento médio das fibras de Calycophyllum spruceanum, Baldin \& Marchiori (2015) encontraram valor aproximado ao obtido no presente estudo, e concluíram que as mesmas representam $47 \%$ do volume da madeira. Os mesmos autores também estudaram a anatomia do lenho de outras duas espécies deste gênero (Calycophyllum candidissimum e Calycophyllum multiflorum), ressaltando que as fibras ocupam 48 e $40 \%$, do volume de madeira, respectivamente.

O comprimento das fibras é influenciado pelas divisões que ocorrem nas direções longitudinais e tangenciais no câmbio (Panshin \& De Zeeuw, 1980), como resultado da taxa de hormônios da árvore, que pode variar com a sazonalidade, características do ambiente, genética e idade da árvore (Alzate, 2004). 
Quanto à largura das fibras, os valores médios nas posições amostradas também diferiram estatisticamente. Os maiores valores foram encontrados nas posições da base e $50 \%$ da altura comercial do tronco. Os valores dos coeficientes de variação foram considerados baixos, sendo menores que $20 \%$.

Para a madeira de paricá, Vidaurre et al. (2018) observaram que a largura das fibras cresceu da base para 50\%, do comprimento do tronco, tendo, a partir desta posição um pequeno decréscimo, sem diferença estatística entre a amostragem realizada a $100 \%$ da altura comercial.

Em relação ao diâmetro do lume, os menores resultados foram observados nas posições do DAP e $25 \%$. Os maiores valores de diâmetro do lume das fibras foram notados nas posições 0, 50, 75 e 100\%. Os coeficientes de variação foram considerados elevados (sendo para a posição 75, superior a 30\%), demonstrando alta variação para esta característica. Florsheim et al. (2009) observaram que os maiores valores de lume para Eucalyptus dunnii, com sete anos de idade, encontram-se na base da árvore.

Entretanto, para a madeira de paricá, Vidaurre et al. (2018) observaram que o diâmetro do lume cresceu da base para 50\%, do comprimento do tronco, tendo, a partir desta posição um pequeno decréscimo, sem diferença estatística entre a amostragem realizada a 100\% da altura comercial.

Em relação à espessura de parede, as posições mais próximas da base foram as que obtiveram maiores valores, corroborando com os estudos de Florsheim et al. (2009) e Gil et al. (2018). Nas posições 75 e 100\% os valores diferiram das demais. No entanto, para três posições ao longo do tronco de angico vermelho (Anadenanthera peregrina), Valente et al. (2013) não observaram diferenças no comprimento, largura, diâmetro de lume e espessura de parede das fibras.

A diminuição do espessamento da parede das fibras no sentido longitudinal observada nesta pesquisa pode ser dada pela maior proporção de madeira juvenil presente no topo das árvores, onde a espessura da parede é menor e o diâmetro do lume das células, maior. A espessura da parede possui relação com a qualidade da madeira. Quanto maior a espessura, maior a massa específica e consequentemente, melhor as propriedades de resistência e rigidez da madeira. A fração parede foi maior nas três posições basais. Os coeficientes de variação foram considerados baixos, sendo inferiores a 17\%.

\section{Características do parênquima radial}

Para a altura do parênquima radial, não houve diferença significativa entre as médias dos tratamentos avaliados (Tabela 3). A espécie possui raios com altura média dentro da faixa obtida por Baldin \& Marchiori (2015), para a mesma espécie em estudo, que variaram de 110-620 $\mu \mathrm{m}$ (unisseriados) e de 200-1230 $\mu \mathrm{m}$ (multisseriados). Os coeficientes de variação referentes a este parâmetro foram considerados elevados. Isso indica muita heterogeneidade no sentindo axial.

Tabela 3 - Valores médios dos parâmetros mensurados dos raios da madeira de Calycophyllum spruceanum no sentido longitudinal, com seis anos de idade.

\begin{tabular}{cccc}
\hline $\begin{array}{c}\text { Posição } \\
(\%)\end{array}$ & $\begin{array}{c}\text { Altura } \\
(\boldsymbol{\mu m})\end{array}$ & $\begin{array}{c}\text { Largura } \\
(\boldsymbol{\mu m})\end{array}$ & $\begin{array}{c}\text { Frequência } \\
\left(\mathbf{n}^{\mathbf{0}} \mathbf{~ m} \mathbf{m}^{-1} \mathbf{)}\right.\end{array}$ \\
\hline 0 & $495,97 \mathrm{a}$ & $42,78 \mathrm{a}$ & $11,68 \mathrm{a}$ \\
& $(29,83)$ & $(34,20)$ & $12,04)$ \\
DAP & $465,22 \mathrm{a}$ & $36,64 \mathrm{~b}$ & $11,11 \mathrm{~b}$ \\
& $(27,46)$ & $(24,29)$ & $12,33)$ \\
25 & $451,58 \mathrm{a}$ & $36,35 \mathrm{~b}$ & $(1,32 \mathrm{a}$ \\
& $(30,40)$ & $(27,14)$ & $10,80 \mathrm{~b}$ \\
50 & $458,36 \mathrm{a}$ & $34,15 \mathrm{~b}$ & $(13,36)$ \\
& $(30,29)$ & $(24,53)$ & $10,77 \mathrm{~b}$ \\
75 & $474,18 \mathrm{a}$ & $38,02 \mathrm{~b}$ & $(14,14)$ \\
& $(30,03)$ & $(25,26)$ & $11,14 \mathrm{~b}$ \\
100 & $446,04 \mathrm{a}$ & $36,82 \mathrm{~b}$ & $(13,32)$ \\
& $(31,85)$ & $(27,58)$ & 11,14 \\
Média Geral & 465,22 & 37,46 & $(12,81)$ \\
\hline
\end{tabular}


$\mathrm{DAP}=$ diâmetro à altura do peito. Valores entre parênteses correspondem ao coeficiente de variação. Médias seguidas pela mesma letra na coluna, não diferem (Scott-Knott, $p>0,05$ ).

Houve diferença entre as médias das posições relacionadas à largura dos raios, com a da posição 0\% superior às demais, corroborando com os estudos de Florsheim et al. (2009). Os elevados coeficientes de variação desse parâmetro indicam que o material possui alta variação em seu tecido radial na direção longitudinal.

Não foi observada uma tendência de variação de frequência dos raios em relação às posições. As médias das posições DAP, 50, 75 e 100\% foram estatisticamente iguais, com menores valores em relação às posições 0 e $25 \%$.

\section{Retratibilidade e massa específica básica}

Para as retratibilidades tangencial e volumétrica, houve diferenças significativas entre as médias das posições no sentido longitudinal (Tabela 4). Os valores foram decrescentes do DAP ao topo da árvore, tendo às contrações das posições 75 e 100\% da altura comercial, sido inferiores às demais. Na contração radial e fator anisotrópico, não houve diferença entre as médias.

Tabela 4 - Valores médios da retratibilidade e massa especifica básica da madeira de Calycophyllum spruceanum no sentido longitudinal, com seis anos de idade.

\begin{tabular}{|c|c|c|c|c|c|}
\hline \multirow{2}{*}{ Posição (\%) } & \multicolumn{3}{|c|}{ Retratibilidade (\%) } & \multirow{2}{*}{$\begin{array}{c}\text { Fator } \\
\text { anisotrópico }\end{array}$} & \multirow{2}{*}{ Massa específica básica $\left(\mathrm{g} \mathrm{cm}^{-3}\right)$} \\
\hline & Tangencial & Radial & Volumétrica & & \\
\hline 0 & $7,29 a$ & $4,04 a$ & $11,34 \mathrm{a}$ & $1,80 \mathrm{a}$ & $0,690 \mathrm{a}$ \\
\hline DAP & $7,45 \mathrm{a}$ & $4,86 a$ & $12,30 \mathrm{a}$ & $1,53 \mathrm{a}$ & $0,652 \mathrm{a}$ \\
\hline 25 & $6,95 \mathrm{a}$ & $4,93 \mathrm{a}$ & $11,88 \mathrm{a}$ & $1,41 \mathrm{a}$ & $0,650 \mathrm{a}$ \\
\hline 50 & $6,82 \mathrm{a}$ & $5,07 a$ & 11,89 a & $1,34 \mathrm{a}$ & $0,616 b$ \\
\hline 75 & $6,25 b$ & $4,51 \mathrm{a}$ & $10,77 \mathrm{~b}$ & $1,39 a$ & $0,606 \mathrm{~b}$ \\
\hline 100 & $6,00 \mathrm{~b}$ & $4,15 a$ & 10,15 b & $1,44 a$ & $0,602 \mathrm{~b}$ \\
\hline Média Geral & $6,79(7,29)$ & $4,59(16,68)$ & $11,39(7,89)$ & $1,49(16,47)$ & $0,636(6,00)$ \\
\hline
\end{tabular}

DAP = diâmetro à altura do peito. Valores entre parênteses correspondem ao coeficiente de variação. Médias seguidas pela mesma letra na coluna, não diferem (Scott-Knott, $p>0,05$ ).

A retratibilidade volumétrica determina a variação total ocorrida na madeira, em função de sua higroscopicidade. Entretanto, as contrações tangenciais e radiais são mais relevantes, pois refletem no coeficiente de anisotropia da madeira. Os valores do coeficiente de anisotropia observados nesta pesquisa foram inferiores aos encontrados por Valente et al. (2013), para a madeira proveniente de sete árvores de angico vermelho, com idade desconhecida, e por Paes et al. (2015) e Gil et al. (2018) para a madeira de teca com sete anos. De acordo com os valores obtidos, a madeira de pau-mulato é considerada boa para utilizações em que se exigem poucas rachaduras e empenamentos, como pisos, móveis e lambris.

Para árvores provenientes de floresta de várzea com DAP entre 18 e $55 \mathrm{~cm}$, obtidas no município de Mazagão - AP, Araújo et al. (2016) obtiveram valores médios de contração tangencial, radial e volumétrica de 7,3; 5,1; e 14,1\%, respectivamente, com fator anisotrópico de 1,6. Valores superiores, porém próximos aos obtidos no presente estudo. Os autores citados constataram o potencial madeireiro da espécie, mesmo proveniente de indivíduos de menores diâmetros (idades).

A massa específica básica da madeira foi próxima da encontrada por Araújo et al. (2016), que obtiveram valor médio de $0,65 \mathrm{~g} \cdot \mathrm{cm}^{-3}$. No presente estudo, os valores decresceram no sentido longitudinal, sendo os maiores encontrados nas posições 0, DAP e $25 \%$ da altura comercial do tronco, diferindo das demais posições. Assim, a madeira jovem de pau-mulo exibiu dois padrões de massa específica, em que da base até a metade do tronco foram maiores e da metade ao topo, menores. 
Já a madeira de Tectona grandis obtida de desbaste, aos 7 anos, estudada por Gil et al. (2018), exibiu maiores valores de massa específica na base do tronco (0\% de altura), redução a $25 \%$ da altura, seguido de aumento até $75 \%$ e decréscimo em direção ao topo da árvore (100\%).

A diminuição dos valores de massa específica básica da madeira de pau-mulato no sentido longitudinal pode ser explicada pela diminuição da espessura da parede das fibras, em que as maiores espessuras foram observadas até 50\% da altura comercial (Tabela 2). Porém, essa variação pode ser alterada com a idade da árvore.

Para a madeira de Calycophyllum spruceanum coletada na Amazônia Peruana, com idade desconhecida, Torre \& Martina (2006) obtiveram valores de 9; 5; e 15\%, nas contrações tangencial, radial e volumétrica, respectivamente. Com fator anisotrópico 1,80 e massa específica básica de 0,76 $\mathrm{g} \mathrm{cm}^{-3}$. Tais valores foram superiores aos encontrados na presente pesquisa e também por Araújo et al. (2016). As diferenças observadas podem estar relacionadas com a idade das árvores, qualidade de sítio e posição amostrada da madeira.

\section{CONCLUSÕES}

Os elementos anatômicos e a massa específica básica da madeira conferem à madeira jovem de pau-mulato (Calycophyllum spruceanum) boas características tecnológicas.

As características macroscópica, microscópicas e as variações longitudinais da massa específica básica da madeira do pau-mulato jovem, proveniente de desbaste, indicam que a mesma poderia ter aplicação industrial em atividades em que não se necessita madeira de grandes dimensões.

Em decorrência da idade e diâmetro das árvores coletadas, as informações sobre a anatomia (macroscópica e microscópica) e características físicas da madeira servem apenas para descrevê-la no estágio em que foram colhidas, e não como uma caracterização geral da espécie.

\section{REFERÊNCIAS}

Alzate, S. B. A. (2004). Caracterização da madeira de árvores de clones de Eucalyptus grandis, E. saligna e E. grandis x urophylla (Tese de doutorado). Escola Superior de Agricultura "Luiz de Queiroz", Universidade de São Paulo, Piracicaba.

Araújo, B. H. P., Sousa, M. A. R., Nascimento, H. E. M., Zanuncio, A. J. V., Rodrigues, D. M. S., \& Guedes, M. C. (2016). Propriedades físicas da madeira de Calycophyllum spruceanum Benth. em função do diâmetro e da posição (base e topo) no fuste. Scientia Forestalis, 44(111), 759-768. http://dx.doi.org/10.18671/scifor.v44n111.22.

Associação Brasileira de Normas Técnicas - ABNT. (1997). NBR 7190: projeto de estruturas da madeira. Anexo B: determinação das propriedades das madeiras para projetos de estruturas. Rio de Janeiro: ABNT.

Associação Brasileira de Normas Técnicas - ABNT. (2003). NBR-11941: madeira: determinação da densidade básica. Rio de Janeiro: ABNT.

Baldin, T., \& Marchiori, J. N. C. (2015). Anatomia da madeira de Calycophyllum spruceanum (Benth.) K. Schum. e Calycophyllum spruceanum F. Brasiliensis K. Schum. Balduinia, (50), 16-23. http://dx.doi.org/10.5902/2358198020325.

Bamber, R. K., Horne, R., \& Graham Higgs, A. (1982). Effect of fast growth on the wood properties of Eucalyptus grandis. Australian Forest Research, 12, 163-167.

Boivin-Chabot, S., Margolis, H. A., \& Weber, J. C. (2004). Variation in coppice-shoot growth among provenances of Calycophyllum spruceanum Benth. in the Peruvian Amazon Basin. Forest Ecology and Management, 198(1-3), 249-260. http://dx.doi.org/10.1016/j.foreco.2004.04.009.

Burger, L. M., \& Richter, H. G. (1991). Anatomia da madeira (154 p.). São Paulo: Nobel.

Campos, J. C. C., \& Leite, H. G. (2002). Mensuração florestal: perguntas e respostas (407 p.). Viçosa: Editora UFV.

Comissión Panamericana de Normas Técnicas - COPANT. (1974). Descripción de características generales, macroscópicas de las maderas angiospermas dicotiledôneas (Vol. 30, pp. 1-19). Buenos Aires: COPANT. 
Florsheim, S. M. B., Couto, H. T. Z., Lima, I. L., \& Longui, E. L. (2009). Variação nas dimensões dos elementos anatômicos da madeira de Eucalyptus dunnii dos sete anos de idade. Revista do Instituto Florestal, 21(1), 79-91.

Gil, J. L. R. A., Barboza, F. S., Coneglian, A., Silva, M. F., Moraes, M. D. A., \& Sette Junior, C. R. (2018). Características físicas e anatômicas da madeira de Tectona grandis L.f. aos 7 anos de idade. Revista de Ciências Agrárias, 41(2), 529-538.

Gomez, F. M., Chagas, M. P., Sette Junior, C. R., \& Tomazello Filho, M. (2006). Caracterização da estrutura anatômica do lenho de árvore de Calycophyllum spruceanum Benth. In Anais eletrônicos do $14^{\circ}$ Simpósio Internacional de Iniciação Científica. São Paulo: USP. Recuperado em 10 de setembro de 2015, de https://uspdigital.usp.br/siicusp/cdOnlineTrabalhoObter?numerolnscricaoTrabnume=4190\&numer oEdicao $=14 \&$ print $=\mathrm{S}$

Guedes, M. C. (2015). Embrapa Amapá desenvolve pesquisas para regeneração natural do pau mulato. Brasília: Embrapa. Recuperado em 10 de fevereiro de 2016, de https://www.embrapa.br/temaintegracao-lavoura-pecuaria-floresta-ilpf/busca-de-noticias/-/noticia/3162581/embrapa-amapadesenvolve-pesquisas-para-regeneracao-natural-do-paumulato

Guedes, M., Castilho, N., Miranda, D., Fischer, G., Sousa, M., Zaharya, N., \& Mochiutt, S. (2012). Regeneração do pau-mulato: manejo para uso sustentável da várzea (2p.). Amapá: Embrapa Amapá. Recuperado em 17 de maio de 2018, de http://ainfo.cnpti a.embrapa.br/digital/bitstream/item/122256/1/CPAF-AP-2012-pau-mulato.pdf

International Association of Wood Anatomists - IAWA. (2007). List of microscopic features for hardwood identification, with an appendix on non-anatomical information. IAWA Bulletin, 3(10), 219-332.

Lorenzi, H. (2002). Árvores brasileiras: manual de identificação e cultivo de plantas arbóreas do Brasil (4. ed.). Nova Odessa: Instituto Plantarum.

Maranho, L. T., Galvão, F., Muñiz, G. I. B., Kuniyoshi, Y. S., \& Preussler, K. (2006). H. Variação dimensional das traqueídes ao longo do caule de Podocarpus lambertii Klotzsch ex Endl., Podocarpaceae. Acta Botanica Brasílica, 20(3), 633-640. http://dx.doi.org/10.1590/S0102-33062006000300013.

Paes, J. B., Santos, L. L., Silva, L. F., Motta, J. P., Braz, R. L., \& Lombardi, L. R. (2015). Caracterização tecnológica da madeira juvenil de teca (Tectona grandis) visando à produção de móveis. Revista Brasileira de Ciências, 10(3), 437-442. http://dx.doi.org/10.5039/agraria.v10i3a3906.

Panshin, A. J., \& De Zeeuw, C. (1980). Textbook of wood technology (4th ed., 705 p.). New York: McGrawHill.

Paula, J. E. (2005). Caracterização anatômica da madeira de espécies nativas do cerrado, visando sua utilização na produção de energia. Cerne, 11(1), 90-100.

Paulino, P. S., \& Lima, I. L. (2018). Densidade básica e dimensões celulares da madeira em clones de Eucalyptus urophylla, aos oito anos de idade. Ciência da Madeira, 9(1), 9-18. http://dx.doi.org/10.12953/2177-6830/rcm.v9n1p9-18.

Pimenta, A. S., Barcellos, D. C., \& Oliveira, E. (2010). Curso de carbonização (94 p.). Viçosa: Universidade Federal de Viçosa, Laboratório de Painéis e Energia da Madeira.

Ramalho, R. S. (1987). O uso de macerado no estudo anatômico de madeiras (4 p.). Viçosa: UFV.

Rocha, F. T., Florsheim, S. M. B., \& Couto, H. T. Z. (2004). Variação das dimensões dos elementos anatômicos da madeira de árvores de Eucalyptus grandis Hill ex Maiden aos sete anos. Revista do Instituto Florestal, 16(1), 43-55. http://dx.doi.org/10.4322/rif.2014.003.

Sotelo Montes, C., Hernández, R. E., Beaulieu, J., \& Weber, J. C. (2007). Genetic variation in wood color and its correlations with tree growth and wood density of Calycophyllum spruceanum at an early age in the Peruvian Amazon. New Forests, 35(1), 57-73. http://dx.doi.org/10.1007/s11056-007-9060-9.

Tomazello Filho, M. (1987). Variação radial da densidade básica e da estrutura anatômica da madeira do Eucalyptus globulus, E. pellita e E. acmenioides. Scientia Forestalis, 36, 35-42.

Torre, C. R., \& Martina, A. M. S. (2006). Guía de procesamiento industrial: fabricación de muebles con maderas poco conocidas - LKS (20 p.). Lima: Argentina S.R.L. Recuperado em 15 de maio de 2016, de http://plantarperu.com/guia_capirona.pdf

Trianoski, R., De Matos, J. L. M., Iwakiri, S., \& Prata, J. G. (2013). Avaliação da estabilidade dimensional de espécies de pinus tropicais. Floresta e Ambiente, 20(3), 398-406.

http://dx.doi.org/10.4322/floram.2012.071. 
Ugarte-Guerra, L. J., \& Domínguez-Torrejón, G. (2010). Índice de sitio (IS) de Calycophyllum spruceanum Benth. en relación con la altura dominante del rodal en ensayos de plantación en la Cuenca del Aguaytía, Ucayali, Perú. Ecología Aplicada, 9(2), 101-111. http://dx.doi.org/10.21704/rea.v9i1-2.400.

Valente, B. M. R. T., Evangelista, W. V., Silva, J. C., \& Della Lucia, R. M. (2013). Variabilidade radial e longitudinal das propriedades físicas e anatômicas da madeira de angico-vermelho. Scientia Forestalis, 41(100), 485-496.

Vidaurre, G. B., Vital, B. R., Colodette, J. L., Oliveira, A. C., Silva, J. G. M., Moulin, J. C., \& Soranso, D. R. (2018). Anatomical and chemical properties of juvenile Schizolobium amazonicum wood. Revista Árvore, 42(3), e420301. http://dx.doi.org/10.1590/1806-90882018000300001.

Contribuição dos autores: JKBA: curadoria de dados, metodologia, investigação, escrita - primeira redação; MDCA: investigação, supervisão, escrita - revisão e edição; JBP: supervisão, escrita - revisão e edição; JTSO: análise formal, supervisão, escrita - primeira redação; SBS: metodologia, investigação; AVF: metodologia, investigação. 